\title{
Stability in Linear Response and Clustering Properties
}

\author{
A. Verbeure and R. A. Weder* \\ University of Leuven, Heverlee, Belgium ${ }^{\star}$
}

Received November 15, 1974

\begin{abstract}
We derive a necessary and sufficient asymptotic condition assuring that a quantum dynamical system in equilibrium is stable in linear response.

We prove, in particular, that if the Hamiltonian has no singular-continuous spectrum and zero is the only eigenvalue, the dynamical system is stable.

Finally we prove that a dynamical system is strongly clustering, if and only if, it is weakly clustering and stable in linear response.
\end{abstract}

\section{Introduction}

In a previous paper [1] we considered an operator representation in a Hilbert space, $\tilde{\mathscr{H}}$, for the response, relaxation and correlation functions for any vector state, $\omega_{0}$, of a von Neumann algebra $\mathfrak{M}$, acting on a Hilbert space $\mathscr{H}$, satisfying the K.M.S. condition, and we proved the existence of the static admittance, and the relaxation. In this paper we apply that technique to the study of the clustering properties of a dynamical system, indicating the usefulness of linear response theory.

Following the ideas of [2] we introduce (Definitions 1 and 2) the notion of stability of a dynamical system under a perturbation of the Hamiltonian, $H_{0}$, by a potential of the type $\lambda V$, where $V$ is any selfadjoint element of $\mathfrak{M}$, and $\lambda$, a real number, is the coupling constant. We derive (Theorem 3 ) an asymptotic condition which is necessary and sufficient to have stability in linear response. We prove, in particular, that if the Hamiltonian has no singular continuous spectrum and zero is the only eigenvalue, the dynamical system is stable in linear response (Theorem 4). Finally we give in Theorem 6 a necessary and sufficient condition for a dynamical system to be strongly clustering, namely: a dynamical system is strongly clustering if and only if it is weakly clustering, and stable in linear response.

Another relation between temporal cluster-properties and dynamical stability for pure thermodynamic phases can be found in Ref. [3].

\section{Stability in Linear Response Theory}

Let us consider an infinite quantum dynamical system in equilibrium, described by a von Neumann algebra, $\mathfrak{M}$, of observables, acting on a Hilbert space, $\mathscr{H}$, and a vectorial state, $\omega_{0}$ :

$$
\omega_{0}(x)=(\Omega, x \Omega), x \in \mathfrak{M},
$$

with $\Omega$ a cyclic element of $\mathscr{H}$.

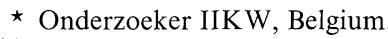

$\star \star$ Postal address: Instituut voor Theoretische Fysica, Celestijnenlaan 200 D, B-3030 Heverlee, Belgium. 
We suppose that $\omega_{0}$ satisfies the K.M.S. condition [4], i.e. it is an equilibrium state. This implies, in particular, that $\Omega$ is separating for $\mathfrak{M}$, and that $\omega_{0}$ is a stationary state:

$$
\omega_{0}\left(\alpha_{t}^{0}(x)\right)=\omega_{0}(x)
$$

where

$$
\alpha_{t}^{0}(x)=e^{i t H_{0}} x e^{-i t H_{0}}, x \in \mathfrak{M},
$$

is the time evolution automorphism. The selfadjoint operator $H_{0}$ is called the Hamiltonian.

The strongly continuous map $t \rightarrow \alpha_{t}^{0}$, is an homomorphism of the additive group of the real line $(\mathbb{R})$ into the *automorphism of $\mathfrak{M}$.

Let us introduce a perturbation of the time evolution automorphism $\alpha_{t}^{0}$, by a selfadjoint potential, $V$, belonging to $\mathfrak{M}$, i.e. we consider a new time evolution automorphism given by

$$
\alpha_{t}(x)=e^{i H t} x e^{-i H t}, x \in \mathfrak{M} ; t \in \mathbb{R},
$$

where the perturbed Hamiltonian, $H$, is equal to $H=H_{0}+\lambda V$, where $V=V^{*} \in \mathfrak{M}$, and $\lambda \in \mathbb{R}$ is the coupling constant. It is easily checked that $\alpha_{t}$ can be developed in a Dyson series,

$$
\begin{gathered}
t \geqq 0 \quad \alpha_{t}(x)=\alpha_{t}^{0}(x)+\sum_{n \geqq 1} i^{n} \lambda^{n} \int d s_{1} \ldots d s_{n}\left[\alpha_{s_{1}}^{0}(v) \ldots,\left[\alpha_{s_{n}}^{0}(v), \alpha_{t}^{0}(x)\right]\right], \\
0 \leqq s_{1} \leqq \ldots \leqq s_{n} \leqq t, x \in \mathfrak{M},
\end{gathered}
$$

and

$$
\begin{aligned}
t \leqq 0 \quad \alpha_{t}(x)= & \alpha_{t}^{0}(x)+\sum_{n \geqq 1}(-i)^{n} \lambda^{n} \int d s_{1} \ldots d s_{n} \\
& \cdot\left[\alpha_{s_{1}}^{0}(v), \ldots,\left[\alpha_{s_{n}}^{0}(v), \alpha_{t}^{0}(x)\right]\right], t \leqq s_{n} \leqq \ldots \leqq s_{1} \leqq 0,
\end{aligned}
$$

$x \in \mathfrak{M}$.

Moreover the series converges absolutely.

We are going to study the evolution given by the Dyson series up to the first order term, i.e. the linear response. In this approximation the perturbed time evolution is equal to:

$$
\alpha_{t}^{v, 1}=\alpha_{t}^{0}(x)+\lambda \int_{0}^{t} d s\left[\alpha_{s}^{0}(v), \alpha_{t}^{0}(x)\right], t \geqq 0, x \in \mathfrak{M},
$$

and

$$
\alpha_{t}^{v, 1}(x)=\alpha_{\tau}^{0}(x)+\lambda \int_{t}^{0} d s\left[\alpha_{s}^{0}(v), \alpha_{t}^{0}(x)\right], t \leqq 0, x \in \mathfrak{M} .
$$

We are interested in the asymptotic behavior of $\alpha_{t}^{v, 1}$ as $t \rightarrow \pm \infty$. In particular we will consider under which conditions the limits $\omega_{ \pm}^{v, 1}(x)=\lim _{t \rightarrow \pm \infty} \omega_{0}\left(\alpha_{t}^{v, 1}(x)\right)$, exist for any $x \in \mathfrak{M}$, and some $V=V^{*} \in \mathfrak{M}$; because the existence of the limits implies that the dynamical system tends, under the perturbation, to a new equilibrium state, in first order:

$$
\omega_{ \pm}^{v, 1}, \text { as } t \rightarrow \pm \infty \text {. }
$$

Moreover if the $\omega_{ \pm}^{v, 1}$ exist it is obvious that

$$
\lim _{\lambda \rightarrow 0} \omega_{ \pm}^{v, 1}(x)=\omega_{0}(x), x \in \mathfrak{M},
$$


i.e. the new equilibrium state tends to the original one as $\lambda \rightarrow 0$, which is in agreement with the intuitive idea of stability.

So that, we define

Definition 1. A dynamical system $\left(\mathfrak{M}, \alpha_{t}^{0}, \omega_{0}\right)$ is said to be stable in linear response under the perturbation $V, V=V^{*} \in \mathfrak{M}$, if the limits

$$
\omega_{ \pm}^{v, 1}(x)=\lim _{t \rightarrow \pm \infty} \omega_{0}\left(\alpha_{t}^{v, 1}(x)\right) \text {, exist for any } \quad x \in \mathfrak{M}
$$

Definition 2. A dynamical system $\left(\mathfrak{M}, \alpha_{t}^{0}, \omega_{0}\right)$ is said to be stable in linear response if it is stable in linear response under any perturbation $V, V=V^{*} \in \mathfrak{M}$.

In [1] we introduced the Hilbert space $\tilde{\mathscr{H}}$, as the closure $\overline{\mathfrak{M}} \sim$ of the von Neumann algebra $\mathfrak{M}$ with respect to the scalar product $(x, y)_{\sim}=(T x \Omega, T y \Omega) ; x, y \in \mathfrak{M}$, where $T=\left(\frac{\Delta-1}{\ln \Delta}\right)^{\frac{1}{2}}, \Delta=\exp (-H)$. It is proved that the operator $U$, defined by $U x=T x \Omega x \in \mathfrak{M}$ extends to a unitary operator from $\tilde{\mathscr{H}}$ into $\mathscr{H}$. Then we denote $\widetilde{H}_{0}=U^{*} H_{0} U$.

Theorem 3. A dynamical system $\left(\mathfrak{M}, \alpha_{t}^{0}, \omega_{0}\right)$ is stable in linear response under the perturbation $V, V=V^{*} \in \mathfrak{M}$, if and only if the sequence of operators $e^{-i t H_{0}} V$ is weakly convergent in $\tilde{\mathscr{H}}$ as $t \rightarrow \pm \infty$ (the asymptotic condition).

Moreover if the $\omega_{ \pm}^{v, 1}$ exist they are equal to $\omega_{ \pm}^{v, 1}(x)=\omega_{0}(x) \mp \lambda\left(x^{*},\left(1-E_{0}^{\sim}\right) V\right)_{\sim}$, where $E_{0}^{\sim}$ is the projector on the null space of $H_{0}^{\sim}$.

Proof. We give the proof for $t \rightarrow+\infty$, the case $t \rightarrow-\infty$ is similar. It follows from the definition of $\alpha_{t}^{v, 1}$ that

$$
\omega_{0}\left(\alpha_{t}^{v, 1}(x)\right)=\omega_{0}(x)+i \lambda \int_{0}^{t} d s \omega_{0}\left(\left[\alpha_{s}^{0}(v), \alpha_{t}^{0}(x)\right]\right), x \in \mathfrak{M} .
$$

By Theorem II.5 of [1] this is equal to

$$
\begin{aligned}
\omega_{0}\left(\alpha_{t}^{v, 1}(x)\right) & =\omega_{0}(x)-\lambda \int_{0}^{t} d s \frac{d}{d s}\left(\alpha_{t}^{0}\left(x^{*}\right), \alpha_{s}^{0}(v)\right)_{\sim} \\
& =\omega_{0}(x)-\lambda\left(x^{*},\left(1-e^{-i t H_{0}}\right) V\right)_{\sim}, t \geqq 0, x \in \mathfrak{M} .
\end{aligned}
$$

Now suppose the system is stable. Then $\left(x, e^{-i t H_{\tilde{o}}} V\right)_{\sim}$ converges when $t \rightarrow+\infty$, for every $x \in \mathfrak{M}$. But as $\mathfrak{M}$ is dense in $\tilde{\mathscr{H}}$ and $e^{-i t H_{\tilde{o}}} V$ is uniformly bounded this implies that the sequence $e^{-i t H_{0}} V$ is weakly convergent when $t \rightarrow+\infty$.

If $e^{-i t H_{0}} V$ is weakly convergent for $t \rightarrow+\infty$, then $\left(1-e^{-i t H \tilde{o}}\right) V$ is also weakly convergent, and the system is obviously stable. Finally suppose that the system is stable, then

$$
\begin{aligned}
\omega_{ \pm}^{v, 1}(x) & =\omega_{0}(x) \mp \lambda \lim _{t \rightarrow \pm \infty}\left(x^{*},\left(1-e^{-i t H \tilde{O}}\right) V\right)_{\sim} \\
& =\omega_{0}(x) \mp \lambda\left(x^{*},\left(1-E_{0}^{\sim}\right) V\right)_{\sim}, x \in \mathfrak{M},
\end{aligned}
$$

because if $w-\lim _{t \rightarrow \pm \infty} e^{-i t H_{0}^{\tilde{O}}} V$ exist it is equal to $E_{0}^{\tilde{0}} V$ by [5].

Theorem 4. Suppose that the Hamiltonian, $H_{0}$, has no singular continuous spectrum and that zero is the only eigenvalue. Then the dynamical system $\left(\mathfrak{M}, \alpha_{t}^{0}, \omega_{0}\right)$ is stable in linear response. 
Proof. As $H_{0}^{\sim}$ is unitary equivalent to $H_{0}$ [1], zero is its only eigenvalue and it has no singular continuous spectrum, then $\widetilde{\mathscr{H}}=\widetilde{\mathscr{H}}_{0}+\widetilde{\mathscr{H}}_{\text {a.c. }}$, where $\widetilde{\mathscr{H}}_{0}$ is the space spanned by the eigenvectors of $H_{0}^{\sim}$, and $\tilde{\mathscr{H}}_{\text {a.c. }}$ is the subspace of absolutely continuous vectors. Hence

$$
V=V_{0} \oplus V_{\text {a.c. }},
$$

and

$$
\left(x^{*}, e^{-i t H_{\tilde{0}}} V\right)_{\sim}=\left(x_{0}^{*}, V_{0}\right)_{\sim}+\left(x_{a c}^{*}, e^{-i t H_{\tilde{0}}} V_{\text {a.c. }}\right)_{\sim},
$$

so that

$\lim _{t \rightarrow \pm \infty}\left(x^{*}, e^{-i t H_{0}^{\tilde{0}}} V\right)_{\sim}=\left(x_{0}^{*}, V_{0}\right)_{\sim}$, where we applied the well known result that $\lim _{t \rightarrow \pm \infty}$ $\left(\mu, e^{-i t \tilde{\sigma}_{\tilde{0}}} v\right)_{\sim}=0$ for any $v \in \tilde{\mathscr{H}}_{a c}$.

Q.E.D.

\section{Stability and Clustering Properties}

Definition 5. A dynamical system $\left(\mathfrak{M}, \alpha_{t}^{0}, \omega_{0}\right)$ is said to be weakly clustering if $\mathfrak{M}_{t} \omega_{0}\left(x \alpha_{t}^{0}(y)\right)=\omega_{0}(x) \omega_{0}(y), x, y \in \mathfrak{M}$,

where $\mathfrak{M}_{t}$ is the mean over $t$; and is said to be strongly clustering if

$$
\lim _{t \rightarrow \pm \infty} \omega_{0}\left(x \alpha_{t}^{0}(y)\right)=\omega_{0}(x) \omega_{0}(y), x, y \in \mathfrak{M} .
$$

In the next Theorem we give a necessary and sufficient condition for a dynamical system to be strongly clustering.

Theorem 6. A dynamical system $\left(\mathfrak{M}, \alpha_{t}^{0}, \omega_{0}\right)$ is strongly clustering if and only if it is weakly clustering and stable in linear response.

Proof. Suppose that the system is strongly clustering. Then it is weakly clustering, and as

$$
\omega\left(x, \alpha_{t}^{0}(v)\right)=\left(x^{*} \Omega, e^{i H_{0} t} V \Omega\right),
$$

the sequence $e^{i H_{0} t} V \Omega$ is weakly convergent as $t \rightarrow \pm \infty$. But this implies that $e^{-i t H_{0}} V$ is weakly convergent as $t \rightarrow \pm \infty$ in $\tilde{\mathscr{H}}$. So that the system is stable, by Theorem 3.

Suppose that the system is stable. Then $e^{-i t H_{0}} y$ is weakly convergent as $t \rightarrow \pm \infty$, for any $y=y^{*} \in \mathfrak{M}$. But as before, this implies that $e^{-i t H_{0}} y \Omega$ is weakly convergent as $t \rightarrow \pm \infty$. Hence

$$
\begin{aligned}
\lim _{t \rightarrow \pm \infty} \omega_{0}\left(x \alpha_{t}^{0}(y)\right) & =\lim _{t \rightarrow \pm \infty}\left(x^{*} \Omega, e^{i t H_{0}} y \Omega\right) \\
& =\left(x^{*} \Omega, E_{0} y \Omega\right), y=y^{*} \in \mathfrak{M} .
\end{aligned}
$$

where $E_{0}$ is the projector on the null space of $H_{0}$.

Suppose in addition that the system is weakly clustering. Then by [6] $E_{0}$ is one dimensional, hence

$$
E_{0} y \Omega=(\Omega, y \Omega) \Omega
$$


and

$\lim _{t \rightarrow \pm \infty} \omega_{0}\left(x \alpha_{t}^{0}(y)\right)=\omega_{0}(x) \omega_{0}(y), x \in \mathfrak{M} ; y=y^{*} \in \mathfrak{M}$

But as any $y \in \mathfrak{M}$, can be decomposed as $y=y_{1}+i y_{2}$, where $y_{1}$ and $y_{2}$ are selfadjoint this is true for any $x, y \in \mathfrak{M}$.

\section{References}

1. Naudts, J., Verbeure, A., Weder, R.: Linear response theory and the KMS condition. Commun. math. Phys. 44, 87-99 (1975)

2. Haag, R., Kastler,D., Trych-Pohlmeyer,E.: Stability and equilibrium states. Commun. math. Phys. 38, 173 (1974)

3. Narnhofer, H., Robinson, D. W.: Dynamical stability and thermodynamic phases. Commun. math. Phys. 41, 89-97 (1975)

4. Haag, R., Hugenholtz, N.M., Winnink, M.: Commun. math. Phys. 5, 215 (1967)

5. Dunford, N., Schwartz, J. T.: Linear operators. Part I, Chapter VIII, 7.1. London: Interscience

6. Knops, H. J.F.: Ergodic states and symmetry breaking in phase transitions, 1969. Thesis Nijmegen, The Netherlands, Theorem II.1.3

A. Verbeure

Institute for Theoretical Physics

Celestijnenlaan $200 \mathrm{D}$

B-3030 Heverlee, Belgium 
